\title{
Neuronal dynamics of sensory coding: the legacy of Jose Pedro Segundo
}

\author{
André Longtin • John Rinzel
}

(C) Springer-Verlag 2009

Neuronal dynamics refers to the temporal behaviors that nervous systems exhibit. By coding we ask what and how does the neuronal activity represent our sensory experience, emotions, mental decisions and actions. A measure of activity or information that ignores temporal structure, say the average over time, gives a zeroth order indication of our world or our interpretation/representation of it. It falls upon on us to reach deeper, to consider the potential for encoding that is offered by the dynamical features of activity. What resources from the brain's wetware enable coding in a dynamical fashion? What are the basic components, the neurons, synapses, circuits dynamically capable of, how do they respond to dynamic stimuli? How does the brain exploit these resources to extract dynamical features and to encode information in spike trains, in multi-unit spatio-temporal activity patterns and across brain areas and via transformations from the periphery to higher associative areas?

A dynamical understanding of brain function builds on knowledge of how systems respond to inputs, in particular, biologically significant input. In the early 1960's, the stimulation paradigm of the day was heavily influenced by the emerging field of cybernetics with its use of linear systems theory. The relevance of this knowledge to true brain function always depends on how well one can relate it to the in vivo situation. From this point of view, sensory systems, along with motor systems, have always presented a distinct advantage because one has a relatively clear idea of what constitutes a relevant input, and what the output is used for-in contrast e.g. to cells buried deep into associative cortices.

\footnotetext{
A. Longtin $(\varangle)$

Ottawa, Canada

e-mail: alongtin@uottawa.ca

J. Rinzel ( $\varangle)$

New York, USA

e-mail: rinzel@cns.nyu.edu
}

Our current understanding of neural activity, from the sub-cellular to the whole brain level, has benefited from the close and cross-disciplinary interaction of practitioners interested in neuronal dynamics - notably, neurophysiologists, and applied mathematicians (or engineers or physicists) versed in the theory of linear/nonlinear dynamics and of time series and point process analysis. It has been particularly advanced by those practitioners who were able to reach out to the "other side", learning enough of each other's language as well as the potential and limitations of each other's approaches. The theorists' approaches can provide, moreover, the conceptual frameworks that enable us to generalize across system levels and that expose, by way of reduction, the mechanistic essence of dynamics in coding.

This special issue on Neuronal Dynamics of Sensory Coding highlights insights that can emerge from attention to temporal aspects of coding. We dedicate the issue to a pioneer in the area, Jose Pedro (Pepe) Segundo, Professor Emeritus at UCLA. Following a bachelor degree in mathematics and premedical sciences, Pepe became a medical doctor. He intended to become a neurosurgeon, but after following a suggestion by a mentor to try postdoctoral work in neurophysiology, as it would be useful, he liked it and never turned back. He strove early on to assimilate emerging concepts in dynamical systems theory and time series/point process analysis in order to apply them to neuroscience. He put his distinct advantage of speaking both "languages" to good use. His work set the stage for much of the growth and synergies that followed in neuronal dynamics and computational neuroscience. Pepe found ways to derive and to illustrate conceptual bases for coding and dynamical repertoires in sensory and other systems with examples and generalizations.

Pepe recognized early on that sensory systems offer opportunities for exploring the role of neuronal dynamics. Much of his early thinking was inspired by the work of Magoun 
and others that targeted the central brain stem areas, then referred to broadly as the "reticular activating system". The extreme convergence and divergence seen in those systems suggested that they performed radically different transformations on data than the more straightforward-looking sensory and motor systems did. It was clear to him that sensory surfaces were mapped (see e.g. Bell et al. 1963) by both unknown spatial and temporal mechanisms, and that accordingly the timing of firing events was likely a significant part of these mechanisms. This set him on the road to unraveling spike train patterns and their functional significance, albeit in simpler, more tractable systems.

Throughout his career he collaborated closely with mathematicians and promptly embraced new concepts and turned them into neurobiological reality. On the time series side, he went beyond characterizing slow large-scale brain rhythms (Segundo et al. 1955; Bell et al. 1963; Buendia et al. 1963), including event-related activity, to focus on the much finer time scales of spiking activity. This led him to point process formulations of such activity. With his collaborators, he developed novel deterministic and stochastic modeling techniques (Moore et al. 1966), including a precursor of the spike response model (Brillinger and Segundo 1979).

Many of the ideas discussed nowadays in neural coding can be found in his seminal paper on the topic (Segundo 1970). The same can be said for the area of spike train analysis, set on firm ground by Segundo and his colleagues (Moore et al. 1966). He and his colleagues were also pioneers in the new field of computational neuroscience (Segundo et al. 1968). He was at the forefront of studies aimed at determining whether the temporal pattern of spikes impinging on a cell may be an important, perhaps the most important, source of information that a neuron must respond to (Segundo et al. 1963, 1966). He thus paid great attention to different firing patterns and their functional implications, including their correlations (Bryant et al. 1973). Soon after, he pioneered the reverse correlation technique and used it both as a modeling technique and to measure the reliability of firing times, especially in the face of noise sources (Bryant and Segundo 1976).

Given the emerging experimental relevance of timing of neural activity, Pepe in parallel went on to, realistically and metaphorically, breathe life into the dynamical phenomena of phase locking, feedback, transients, noise and multi-stability. In their seminal paper, they elucidated the effects of repeated stimuli on neural firing, i.e. phase locking (Perkel et al. 1964). He looked at the effects of variability in the presynaptic activity on phase locking (Kohn et al. 1981). He explored the world of feedback, and its consequences on neural coding and nonlinear phenomena such as multistability (Vibert et al. 1979; Diez Martinez and Segundo 1983). He investigated theoretically and experimentally the dynamics of coupled pacemaker neurons (Segundo et al. 1987). And he emphasized the importance of investigating transient phenomena to get at the core of neural coding (Segundo et al. 1993). His longstanding reflections on dynamical aspects of spike train genesis and coding can be found in his review (Segundo 2003).

Many signposts of Pepe's rich trajectory of research from novel clinical work on brain responses through the study of dynamics of simple sensory systems have appeared in Kybernetik/Biological Cybernetics. We thus felt it important to recognize these pioneering efforts and dedicate to him this special issue on the theme of Neuronal Dynamics of Sensory Coding, the final issue of Biological Cybernetics" Volume 100 .

This issue brings together recent novel work and review work from modelers and experimentalists in this area. Pepe's work spanned the properties of many preparations, from invertebrates to cat to monkey and man, and accordingly the papers cover a range of preparations as well. Interestingly, some contributors have been trained in both areas, and this ability to pursue a career on both fronts is a very real part of Segundo's legacy. Some of the work in this issue was presented at the 2007 Neural Coding meeting, to which Pepe has contributed greatly over the years, and which was held that year in Montevideo and in his honor.

The contributors to this issue carry on research on different aspects of sensory dynamics including its relation to information theory, memory and motor systems. While in no way is it comprehensive, we believe it is representative of the ongoing efforts in this field.

Today we think naturally of framing questions about neural activity in dynamical terms. We ask what the underlying dynamics are, i.e. what are the linearities, the nonlinearities and bifurcations, how these respond to input that is correlated in time and space, as well as with stochastic inputs with varying degrees of autocorrelation and cross-correlation. We ask what dynamical mechanisms underlie any ideas about coding.

In the early days of Pepe's career however, the idea of performing dynamical analysis of brain activity, beyond the standard systems identification approach advocated by cybernetics, was very novel and still somewhat inchoate, and Pepe was one of the main pioneers into this strange new land. Indeed, we are reminded of the famous quip of the Argentine author Jose Luis Borges (1974), which Pepe liked to paraphrase by saying that a map of a country that was the size of the country itself and that point by point coincided with it is useless to future generations. Debates over the proper level of detail to include in mathematical models of neural data and brain function still rage on, and have much to do about the "size of maps". Pepe, through his extensive seminal contributions to dynamical neuroscience on the experimental, modeling and computational fronts, and his exquisite ability (to this day) to bring together people with complementary 
expertise to focus on specific problems, was able to draw the outline of the dynamical brain map that we all strive to perfect to this day.

\section{References}

Bell C, Buendía N, Sierra G, Segundo JP (1963) Mesencephalic reticular responses to natural and to repeated sensory stimuli. Experientia 19:308-312

Borges JL (1974) Del rigor en la Ciencia, Obras completas, vol II, p 225 Emecé Editores, Buenos Aires, Edición dirigida y realizada por Frías, C.V

Brillinger DR, Segundo JP (1979) Empirical examination of the threshold model of neuron firing. Biol Cybern 35:213-220

Bryant HL Jr, Ruiz Marcos A, Segundo JP (1973) Correlations of neuronal spike discharges produced by monosynaptic connections and by common inputs. J Neurophysiol 36:205-225

Bryant HL, Segundo JP (1976) Spike initiation by transmembrane current: a white-noise analysis. J Physiol (Lond) 260:279-314

Buendía N, Goode M, Sierra G, Segundo JP (1963) Responsiveness and discrimination during sleep. Experientia 19:208-211

Diez Martínez O, Segundo JP (1983) Behavior of a single neuron in a recurrent excitatory loop. Biol Cybern 47:33-41

Kohn AF, Freitas da Rocha A, Segundo JP (1981) Presynaptic irregularity and pacemaker inhibition. Biol Cybern 41:5-18

Moore GP, Perkel DP, Segundo JP (1966) Statistical analysis and functional interpretation of neuronal spike data. Ann Rev Physiol $28: 493-522$
Perkel DP, Schulman J, Bullock TH, Moore GP, Segundo JP (1964) Pacemaker neurons: effects of regularly spaced synaptic input. Science 145:61-63

Segundo JP, Arana R, French JD (1955) Behavioral arousal by stimulation of the brain in the monkey. J Neurosurg 12:601-613

Segundo JP, Moore GP, Stensaas L, Bullock TH (1963) Sensitivity of neurons in Aplysia to temporal patterns of arriving impulses. J Exp Biol 40:643-667

Segundo JP, Perkel DP, Moore GP (1966) Spike probability in neurons: influence of temporal structure in the train of synaptic events. Kybernetik 3:67-82

Segundo JP, Perkel DP, Wyman H, Hegstad H, Moore GP (1968) Inputoutput relations in computer-simulated nerve cells Influence of the statistical properties, number and interdependence of excitatory pre-synaptic terminals. Kybernetik 4:157-171

Segundo JP (1970) Communication and coding by nerve cells. In: Quarton GC, Melnechuk T, Schmitt FO (eds) The neurosciences, second study program. Rockefeller University Press, New York, pp 569-586

Segundo JP, Diez Martínez O, Quijano H (1987) Testing a model of excitatory interaction between pacemakers. Biol Cybern 55:355365

Segundo JP, Stiber M, Altshuler E, Vibert J-F (1993) Transients in the inhibitory driving of neurons and their postsynptic consequences. Neurosci 62:459-480

Segundo JP (2003) Nonlinear dynamics of point process systems and data. Int J Bifurc Chaos 13(8):2035-2116

Vibert JF, Davis M, Segundo JP (1979) Recurrent inhibition. Its influence upon transduction and afferent discharges in slowly adapting stretch receptor organs. Biol Cybern 31:167-178 